\title{
The Analysis of Fractional-Order Navier-Stokes Model Arising in the Unsteady Flow of a Viscous Fluid via Shehu Transform
}

\author{
Pongsakorn Sunthrayuth $\mathbb{D}^{1},{ }^{1}$ Rasool Shah $\mathbb{D}^{2},{ }^{2}$ A. M. Zidan $\mathbb{D}^{3,4}$ Shahbaz Khan, ${ }^{2}$ \\ and Jeevan Kafle $\left.{ }^{5}\right)^{5}$ \\ ${ }^{1}$ Department of Mathematics and Computer Science, Faculty of Science and Technology, Rajamangala University of Technology \\ Thanyaburi (RMUTT), Thanyaburi, Pathumthani, Thailand \\ ${ }^{2}$ Department of Mathematics, Abdul Wali Khan University, Mardan 23200, Pakistan \\ ${ }^{3}$ Department of Mathematics, College of Science, King Khalid University, P.O. Box: 9004, Abha 61413, Saudi Arabia \\ ${ }^{4}$ Department of Mathematics, Faculty of Science, Al-Azhar University, Assuit, 71511, Egypt \\ ${ }^{5}$ Central Department of Mathematics, Tribhuvan University, Kritipur, Kathmandu, Nepal
}

Correspondence should be addressed to Jeevan Kafle; jeevan.kafle@cdmath.tu.edu.np

Received 30 April 2021; Accepted 22 July 2021; Published 10 August 2021

Academic Editor: Nehad Ali Shah

Copyright (C) 2021 Pongsakorn Sunthrayuth et al. This is an open access article distributed under the Creative Commons Attribution License, which permits unrestricted use, distribution, and reproduction in any medium, provided the original work is properly cited.

This paper presents a new method that is constructed by combining the Shehu transform and the residual power series method. Precisely, we provide the application of the proposed technique to investigate fractional-order linear and nonlinear problems. Then, we implemented this new technique to obtain the result of fractional-order Navier-Stokes equations. Finally, we provide threedimensional figures to help the effect of fractional derivatives on the actions of the achieved profile results on the proposed models.

\section{Introduction}

Fractional calculus is an engineering and sciences area that deals with the integral and derivative of arbitrary orders. Fractional differential equations (FDEs) have gained popularity and significance, primarily due to their proven implementations in applied mathematics. For instance, these problems are more and more utilized to an equation in fluid flow, diffusion, rheology, oscillation, relaxation, reaction-diffusion, anomalous diffusion, diffusive transport akin to diffusion, turbulence, polymer physics, electric networks, electrochemistry of corrosion, chemical physics, propagation of seismic waves, relaxation processes in complex systems, porous structures, and dynamical processes in self-similar and various other physical procedure. The most significant benefit of FDEs in these and other uses is their nonlocal property. It is well-known that the differential operator of integer-order is a local operator, but the fractional-order differential operator is nonlocal [1-5]. For example, the nonlinear oscillation of earthquakes can be modeled with fractional derivatives [6], and the fluid dynamic traffic model with fractional derivatives [7] can calculate the insufficiency arising from the assumption of continuum traffic flow. Experimental data fractional partial differential equations for seepage flow in porous media are shown in [8]. Fractional differential equations have created attention among the researcher due to the exact description of nonlinear phenomena, especially in nanohydrodynamics where continuum assumption does not well, and fractional model can be considered the best candidate. These findings invoked the growing interest in studies of fractal calculus in many branches of engineering and science. This is more realistic and it is one reason why fractional calculus has become more and more popular [9-17].

A famous governing equation of motion of viscous fluid flow called the Navier-Stokes (NS) equation has been derived in 1822 [18]. The equation can be regarded as the Momentum equation and is a combination of Newton's second law of motion for fluid substances, the energy equation, and continuity equation. This equation describes many physical things such as ocean currents, liquid flow in pipes, blood 
flow, and airflow around the wings of an aircraft. The NSE is the computational fluid dynamics initial equation, relating external forces and pressure acting on a fluid to the fluid flow response. The Navier-Stokes and continuity models are described by

$$
\left\{\begin{array}{l}
D_{\eta}^{\omega} u+(u . \nabla) u=-\frac{1}{\rho} \nabla p+v \nabla^{2} u, 0<\omega \leq 1, \\
\nabla u=0
\end{array}\right.
$$

where the Caputo fractional derivative is $D_{\eta}^{\infty} u$, time is $\eta$, the velocity vector is $u$, kinematics viscosity is $v$, pressure is $p$, and density is $\rho$. In this article, we consider two special cases. First, a viscous fluid in a tube moves in an unsteady, onedimensional motion. The fluid flow in the tube of fractional NSEs in cylindrical coordinates that govern is defined by

$$
D_{\eta}^{\omega} u+P+v\left(\frac{\partial^{2} u}{\partial \zeta^{2}}+\frac{1}{\zeta} \frac{\partial u}{\partial \zeta}\right), 0<\omega \leq 1,
$$

with initial condition

$$
u(\zeta, 0)=g(\zeta)
$$

where $P=-1 / \rho(\partial p / \partial z)$ and $g(\zeta)$ is a function that depends only on $\zeta$.

Consider the two-dimensional fractional NSE is given as

$$
\begin{aligned}
& D_{\eta}^{\oplus} u=\rho_{0}\left(\frac{\partial^{2}}{\partial \zeta^{2}} u+\frac{\partial^{2}}{\partial \varphi^{2}} u\right)-u \frac{\partial}{\partial \zeta} u-v \frac{\partial}{\partial \varphi} u+g, \\
& D_{\eta}^{\oplus} v=\rho_{0}\left(\frac{\partial^{2}}{\partial \zeta^{2}} v+\frac{\partial^{2}}{\partial \varphi^{2}} v\right)-u \frac{\partial}{\partial \zeta} v-v \frac{\partial}{\partial \varphi} v-g
\end{aligned}
$$

with initial conditions

$$
u(\zeta, \varphi, \eta)=f(\zeta, \varphi), v(\zeta, \varphi, \eta)=g(\zeta, \varphi)
$$

where $u=u(\zeta, \varphi, \eta), v=v(\zeta, \varphi, \eta)$, where time, pressure and constant density define by $\eta, p$, and $\rho$, respectively. $\zeta$ and $\varphi$ are the spatial components and $f(\zeta, \varphi)$ and $g(\zeta, \varphi)$ are two functions dependent only on $\zeta$ and $\varphi$. El-Shahed and Salem [19] generalized the classical NSEs by substituting a Caputo fractional derivative of order $\omega$ for the first time derivative, where $0<\omega \leq 1$. Momani and Odibat [20] use the Adomian decomposition approach to solve fractional-order NSEs. Ganji et al. [21] used the Homotopy perturbation procedure, an analytical technique, to solve fractional NSEs in polar coordinates, and the solutions were given in the closedform. Singh and Kumar [22] implemented the reduced differential transform method to obtain an analytical solution of fractional multidimensional NSEs. Ahmad and Jaber used the residual power series approach to solve the nonlinear fractional two-dimension NSEs [23]. For a class of NSEs with fractional-order derivatives, Wang and Zhang [24] proposed a numerical method. Some investigators combined two strong methods to produce a new method for solving fractional-order NSE equations. Below, we expressed some of these combinations: a mixture of the Adomian decomposition method and Laplace transform; Kumar et al. [25] introduce homotopy perturbation transformation technique, combine the Homotopy perturbation technique with Laplace transform, to solve fractional NSEs. Jena and Chakraverty [26] applied the homotopy perturbation transform technique and this method consists in the combination of homotopy perturbation technique and Elzaki transform; Prakash et al. [27] proposed q-homotopy analysis transform method to obtain a solution of fractional system NSEs. This method combined the homotopy analysis technique and Laplace transform.

The residual power series method (RPSM) is an efficient and straightforward method for constructed a Power Series solution for extremely linear and nonlinear equations without linearization, perturbation, and discretization. The RPS method, unlike the classical power series method, does not involve comparing the coefficients of the corresponding terms or a recursion relation. The power series coefficients are calculated using a series of algebraic equations in one or more variables. The methodology's key benefit is its dependence on derivation, which is much simpler and more accurate than integration, which is the basis of most other solutions techniques. This approach is an alternative technique for solving fractional-order partial differential equations theoretically [28].

The RPSM was used as an essential tool for determining power series method values for fuzzy differential equations [29]. It has been successes used in the analytical solution of the generalize Lane-Emden equations [30], which is significantly nonlinear singular differential equations, in the exact work of higher-order regular differential equations [31], in the result of composite and noncomposite time-fractional differential equations [32], in estimating and demonstrating the variety of solutions for fractional boundary value equation [33], in the approximate investigation of the nonlinear time-fractional Burgers and $\mathrm{KdV}$ equations [34], in further to few other implementations [35-37], and recently, it has been implemented to investigate the analytical solution of a fractional two-component evolutionary system [38]. This paper presents an analytical investigation of one- and twodimensional, fractional-order model of Navier-stokes equations by using RPSTM.

The fractional calculus fundamental definitions are defined in Section 2, write the general methodologies in Section 3, and many test models to show the effectiveness of suggested techniques are given in Section 4, and finally, the conclusion is given in Section 5 .

\section{Basic Definitions}

2.1. Definition. The fractional-order Riemann-Liouville integral is defined as $[39,40]$.

$$
I_{0}^{\delta} h(\eta)=\frac{1}{\Gamma(\delta)} \int_{0}^{\eta}(\eta-s)^{\delta-1} h(s) d s
$$


2.2. Definition. The fractional-order Caputo's derivative of $f(\eta)$ is given as $[39,40]$.

$$
D_{\eta}^{\delta} f(\eta)=I^{j-\delta} f^{j}, j-1<\delta<j, j \in \mathbb{N} \frac{d^{j}}{d \eta^{j}} h(\eta), \delta=j, j \in \mathbb{N} .
$$

2.3. Definition. Shehu transformation is new and identical to other integral transformations defined for exponential order functions. In Set A, the function is defined by [41-43]

$$
A=\left\{v(\eta): \exists, \rho_{1}, \rho_{2}>0,|v(\eta)|<M e^{\frac{|\eta|}{\rho_{i}}} \text {, if } \eta \in[0, \infty) .\right.
$$

The Shehu transform which is described as $S($.) for a function $v(\eta)$ is defined as

$$
S\{v(\eta)\}=V(s, u)=\int_{0}^{\infty} e^{\frac{-s \eta}{u}} v(\eta) d \eta, \eta>0, s>0 .
$$

The Shehu transform of a function $v(\eta)$ is $V(s, u)$; then, $v(\eta)$ is called the inverse of $V(s, u)$ which is given as

$$
S^{-1}\{V(s, u)\}=v(\eta) \text {, for } \eta \geq 0, S^{-1} \text { is inverse Shehu transform. }
$$

2.4. Definition. Shehu transform for $n$th derivatives is given as [41-43]

$$
S\left\{v^{(j)}(\eta)\right\}=\frac{s^{j}}{u^{j}} V(s, u)-\sum_{k=0}^{j-1}\left(\frac{s}{u}\right)^{j-k-1} v^{(k)}(0)
$$

2.5. Definition. The fractional-order derivatives of Shehu transformation are defined as [41-43]

$$
S\left\{v^{(\delta)}(\eta)\right\}=\frac{s^{\delta}}{u^{\delta}} v(s, u)-\sum_{k=0}^{j-1}\left(\frac{s}{u}\right)^{\delta-k-1} v^{(k)}(0), 0<\beta \leq n
$$

\subsection{Definition. A power series is given as}

$$
\sum_{k=0}^{\infty} P_{k}(\eta-\zeta)^{k \oplus}=P_{o}+P_{1}(\eta-\zeta)+P_{2}(\eta-\zeta)^{2 \oplus}+\cdots
$$

where $0 \leq k-1<\omega \leq k$ and $\eta \leq \zeta$ is called fractional-order power series about $\zeta$, where $P_{k}$ are the constant called the coefficient of the series. If $\zeta=0$, then the fractional-order power series will be reduced to the fractional-order Maclaurin series.

2.7. Theorem. Suppose that $f_{o}$ has a fractional power series expression of the form at tau $=\mathrm{psi}$

$$
f_{o}(\eta)=\sum_{m=0}^{\infty} P_{m}(\eta-\zeta)^{m \varpi}
$$

If $D^{m \omega} f_{o}(\eta)$, for $m=0,1,2,3, \cdots$ are continuous on $(\zeta, \zeta$ $+R_{o}$ ), then the coefficient $P_{m}$ can be defined as

$$
P_{m}=\frac{D^{m \varpi} f_{o}(\zeta)}{\Gamma(1+m \omega)}
$$

2.8. Definition. The power series expansion is given as

$$
\sum_{m=0}^{\infty} G_{m}(\phi)(\eta-\zeta)^{m \omega}
$$

is called to be multi fractional power series at $\eta=\zeta$, where $G_{m}(\phi)$ are the fractional power series coefficients.

2.9. Theorem. Let us suppose that $u_{o}(\phi, \eta)$ has the fractionalorder power define at $\eta=\zeta$ is given as

$$
u_{o}(\phi, \eta)=\sum_{m=0}^{\infty} G_{m}(x)(\eta-\zeta)^{m \omega}
$$

If $D_{\eta}^{m \omega} u_{o}(\phi, \eta), m=0,1,2,3, \cdots$ are the continuous on $I_{o}$ $\times\left(\zeta, \zeta+R_{o}\right)$, then the coefficient $G_{m}$ can be written as

$$
G_{m}(x)=\frac{D_{\eta}^{m \omega} u_{o}(\phi, \eta)}{\Gamma(1+m \omega)},
$$

where $\zeta \in I_{o}, \zeta \leq \eta<\zeta+R_{o}, R_{o}$.

So we can write the fractional power expansion of $u_{o}(\phi$,

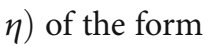

$$
\mu(\phi, \eta)=\sum_{m=0}^{\infty} \frac{D_{\eta}^{m \omega} \mu(\phi, \eta)}{\Gamma(1+m \omega)}(\eta-\zeta)^{m \omega}
$$

which is the generalized form of the Taylor expansion.

2.10. Corollary. Let assume that $\mu(\phi, \varphi, \eta)$ has a power series multi fractional represent about $\eta=\zeta$ as

$$
u_{o}(\phi, \varphi, \eta)=\sum_{m=0}^{\infty} G_{m}(\phi, \varphi)(\eta-\zeta)^{m \varpi}
$$

If $D_{\eta}^{m \omega} \mu(\phi, \varphi, \eta), m=0,1,2,3$.. are continuous on $I_{1} \times$ $I_{2} \times\left(\zeta, \zeta+R_{o}\right)$, then

$$
G_{m}(\zeta, \varphi)=\frac{D_{\eta}^{m \varpi} \mu(\phi, \varphi, \eta)}{\Gamma(1+m \omega)},
$$

where $(\phi, \varphi) \in I_{1} \times I_{2}, \zeta \leq \eta<\zeta+R_{o}$.

\section{The General Methodologies of RPSTM}

In this section, we will go through the steps that RPSTM takes to solve the FPDEs.

$$
D_{\eta}^{\omega} u(\zeta, \eta)=a D_{\zeta}^{2} u(\zeta, \eta)+b u(\zeta, \eta)-c u^{q}(\zeta, \eta)
$$


with initial condition

$$
u(\zeta, 0)=f_{0}(\zeta)
$$

we use the Shehu transformation to (22)

$$
S\left[D_{\eta}^{\infty} u(\zeta, \eta)\right]=a S\left[D_{\zeta}^{2} u(\zeta, \eta)\right]+b S[u(\zeta, \eta)]-c S\left[u^{q}(\zeta, \eta)\right] .
$$

By the fact that $S\left[D_{\eta}^{\infty} u(\zeta, \eta)\right]=\left(s^{\infty} / v^{\Phi}\right) S[u(\zeta, \eta)]-\left(v^{1-\Phi} /\right.$ $\left.s^{1-\Phi}\right) u(x, o)$ and apply the initial condition (23), we can write $(10)$ as

$$
U(\zeta, s)=\frac{v}{s} f_{0}(\zeta)+\frac{v^{\omega}}{s^{\omega}} a D_{x}^{2} U(\zeta, s)+b \frac{v^{\infty}}{s^{\omega}} U(\zeta, s)-c \frac{v^{\infty}}{s^{\omega}} s^{-1}\left[(S[U(\zeta, s)])^{q}\right],
$$

where $U(\zeta, s)=S[u(\zeta, \eta)]$.

Second, we expressed the transformation function $U(\zeta, s)$ as the following equation

$$
U(\zeta, s)=\sum_{n=0}^{\infty}\left(\frac{\nu}{s}\right)^{n \oplus+1} f_{n}(x) .
$$

The $k$ th-truncated series of (26), we write as

$$
U(\zeta, s)=\sum_{n=0}^{\infty}\left(\frac{\nu}{s}\right)^{n \oplus+1} f_{n}(x)=\frac{v}{s} f_{0}(x)+\sum_{n=1}^{\infty}\left(\frac{\nu}{s}\right)^{n \oplus+1} f_{n}(x) .
$$

As stated in [24], the definition of Shehu residual function to (26) is

$$
\begin{aligned}
\operatorname{SRes}(\zeta, s) & =U(\zeta, s)-f_{0}(x) \frac{v}{s}-a \frac{v^{\Phi}}{s^{\omega}} D_{x}^{2} U(\zeta, s)-b \frac{v^{\Phi}}{s^{\omega}} U(\zeta, s) \\
& +c \frac{v^{\Phi}}{s^{\omega}} S^{-1}\left[(S[U(\zeta, s)])^{q}\right],
\end{aligned}
$$

and the $k$ th-Shehu residual function of (28) is

$$
\begin{aligned}
\operatorname{SRes}(\zeta, s)= & U_{k}(\zeta, s)-f_{0}(x) \frac{\nu}{s}-a \frac{v^{\omega}}{s^{\omega}} D_{x}^{2} U_{k}(\zeta, s) \\
& -b \frac{v^{\omega}}{s^{\omega}} U_{k}(\zeta, s)+c \frac{v^{\omega}}{s^{\omega}} S\left[\left(S^{-1}\left[U_{k}(\zeta, s)\right]\right)^{q}\right],
\end{aligned}
$$

Third, we explain some properties of the residual power series method

(i) $\operatorname{SRes}(\zeta, s)=0$ and $\lim _{k \rightarrow \infty} \operatorname{RRes}_{k}(\zeta, s)=\operatorname{SRes}(\zeta, s)$ for each $s>0$

$$
\lim _{k \longrightarrow \infty} \operatorname{SRes}(\zeta, s)=0 \Longrightarrow \lim _{k \longrightarrow \infty} s \operatorname{Res}{ }_{k}(\zeta, s)=0
$$

(ii) $\lim _{k \longrightarrow \infty} s^{k \omega+1} \operatorname{Res}(\zeta, s)=\lim _{k \longrightarrow \infty} s^{k \omega+1} \operatorname{Res}_{k}(\zeta, s)=$ $0,0<\omega \leq 1, k=1,2,3 \cdots$

Furthermore, to calculate the coefficient function $f_{n}(\zeta)$, the following system can be solved recursively.

$$
\lim _{s \longrightarrow \infty}\left(s^{k \omega+1} \operatorname{SRes}_{k}(\zeta, s)\right)=0,0<\omega \leq 1, k=1,2,3, \cdots
$$

Finally, we used the Shehu inverse to $U_{k}(\zeta, s)$, to obtain the $k$ th-approximated supportive result $u_{k}(\zeta, \eta)$.

\section{Numerical Result}

4.1. Example. Consider the one dimensional fractional NS equation

$$
D_{\eta}^{\infty} u(\zeta, \eta)=\frac{\partial^{2} u}{\partial \zeta^{2}}+\frac{1}{\zeta} \frac{\partial u}{\partial \zeta}, 0<\omega \leq 1
$$

Subject to the initial condition

$$
u(\zeta, 0)=\zeta .
$$

Applying Shehu transform to Eq. (32) and using the initial condition given in Eq. (33), we get

$$
U(\zeta, s)=\frac{v}{s}(\zeta)+\frac{v^{\infty}}{s^{\omega}} S_{\eta}\left[S_{\eta}^{-1}\left\{\frac{\partial^{2}}{\partial \zeta^{2}} U(\zeta, s)\right\}\right]+\frac{v^{\infty}}{s^{\infty}} S_{\eta}\left[S_{\eta}^{-1}\left\{\frac{1}{\zeta} \frac{\partial}{\partial x} U(\zeta, s)\right\}\right] .
$$

The $k$ th truncated term series of Eq. (34) is

$$
U_{k}(\zeta, s)=\frac{\nu}{s}(\zeta)+\sum_{n=1}^{k}\left(\frac{\nu}{s}\right)^{n \oplus+1} f_{n}(\zeta)
$$

and the $k$ th Shehu residual function is

$$
\begin{aligned}
S_{\eta} \operatorname{Res}_{k}= & U_{k}(\zeta, s)-\frac{v}{s}(\zeta)-\frac{v^{\omega}}{s^{\omega}} S_{\eta}\left[S_{\eta}^{-1}\left\{\frac{\partial^{2}}{\partial \zeta^{2}} U_{k}(\zeta, s)\right\}\right] \\
& -\frac{v^{\infty}}{s^{\omega}} S_{\eta}\left[S_{\eta}^{-1}\left\{\frac{1}{\zeta} \frac{\partial}{\partial x} U_{k}(\zeta, s)\right\}\right] .
\end{aligned}
$$

Now, to determine $f_{k}(\zeta), k=1,2,3, \cdots$, we put the $k$ thtruncated series (35) into the $k$ th-Shehu residual function (36), multiply the solution equation by $s^{k \omega+2}$ and, then, solve recursively the relation $\lim _{s \longrightarrow \infty}\left[(v / s)^{n \omega+1} \operatorname{Res}_{k}(\zeta, s)\right]=0, k$ $=1,2,3, \cdots$ for $f_{k}(\zeta)$. The first few component of the series 
$f_{k}(\zeta, \varphi)$

$$
\begin{gathered}
f_{1}(\zeta)=\frac{1}{\zeta}, \\
f_{2}(\zeta)=\frac{1}{\zeta^{3}}, \\
f_{3}(\zeta)=3^{3} \frac{1}{\zeta^{5}}, \\
f_{4}(\zeta)=5^{2} \frac{1}{\zeta^{7}}
\end{gathered}
$$

Putting the values of $f_{n}(\zeta)(n \geq 1)$ in Eq. (35), we get

$U(\zeta, s)=\frac{v}{s}\left(1-\zeta^{2}\right)+\frac{v^{\omega}}{s^{\omega}} f_{1}(\zeta)+\frac{v^{\omega+1}}{s^{\omega+1}} f_{2}(\zeta)+\frac{v^{\omega+2}}{s^{\omega+2}} f_{3}(\zeta)+\cdots$,

$$
U(\zeta, s)=\frac{v}{s}(\zeta)+\frac{v^{\Phi}}{s^{\omega}} \frac{1}{\zeta}+\frac{v^{\omega+1}}{s^{\omega+1}} \frac{1}{\zeta^{3}}+\frac{v^{\omega+2}}{s^{\omega+2}} \frac{3^{2}}{\zeta^{5}}+\cdots
$$

Applying inverse Shehu transform to Eq. (39), we get

$u(\zeta, \eta)=\zeta+\frac{1}{\zeta} \frac{\eta^{\Phi}}{\Gamma(\omega+2)}+\frac{1}{\zeta^{3}} \frac{\eta^{2 ळ}}{\Gamma(2 \omega+2)}+\frac{3^{2}}{\zeta^{5}} \frac{\eta^{3 \omega}}{\Gamma(3 \omega+2)}+\cdots$

The above equation put $₫=1$, we get

$$
\begin{gathered}
u(\zeta, \eta)=\zeta+\frac{1}{\zeta} \eta+\frac{1}{\zeta^{3}} \frac{\eta^{2}}{2 !}+\frac{3^{2}}{\zeta^{5}} \frac{\eta^{3}}{3 !}+\cdots \\
u(\zeta, \eta)=\zeta+\sum_{n=1}^{\infty} \frac{1^{2} \times 3^{2} \times 5^{3} \times \cdots \times(2 n-3)^{2}}{r^{2 n-1}} \frac{\eta^{n}}{n !}
\end{gathered}
$$

which is the actual result of classical Navier-Stokes equations for the velocity field. The field of velocity behavior of the classical Navier-Stokes equations is shown in Figure 1, and the fractional-order behavior of Navier-Stokes equations with $\emptyset$ $=0.8,0.6$ and 0.4 is shown in Figures 2 and 3, respectively.

4.2. Example. Consider the one-dimensional fractional NS equation

$$
D_{\eta}^{\Phi} u(\zeta, \eta)=P+\frac{\partial^{2} u}{\partial \zeta^{2}}+\frac{1}{\zeta} \frac{\partial u}{\partial \zeta}, 0<\omega \leq 1
$$

Subject to the initial condition

$$
u(\zeta, 0)=1-\zeta^{2}
$$

Applying Shehu transformation to Eq. (42) and applying the initial condition given in Eq. (43), we get

$$
\begin{aligned}
U(\zeta, s)= & \frac{v}{s}\left(1-\zeta^{2}\right)+\frac{v^{\infty}}{s^{\omega}} S_{\eta}[P]+\frac{v^{\infty}}{s^{\omega}} S_{\eta}\left[S_{\eta}^{-1}\left\{\frac{\partial^{2}}{\partial \zeta^{2}} U(\zeta, s)\right\}\right] \\
& +\frac{v^{\infty}}{s^{\omega}} S_{\eta}\left[S_{\eta}^{-1}\left\{\frac{1}{\zeta} \frac{\partial}{\partial \zeta} U(\zeta, s)\right\}\right] .
\end{aligned}
$$

The $k$ th truncated term series of Eq. (44) is

$$
U_{k}(\zeta, s)=\frac{\nu}{s}\left(1-\zeta^{2}\right)+\sum_{n=1}^{k}\left(\frac{\nu}{s}\right)^{n \oplus+1} f_{n}(\zeta)
$$

and the $k$ th Shehu residual function is

$$
\begin{aligned}
S_{\eta} \operatorname{Res}_{k}= & U_{k}(\zeta, s)-\frac{v}{s}\left(1-\zeta^{2}\right)-\frac{v^{\infty}}{s^{\infty}} P-\frac{v^{\infty}}{s^{\infty}} S_{\eta}\left[S_{\eta}^{-1}\left\{\frac{\partial^{2}}{\partial \zeta^{2}} U_{k}(\zeta, s)\right\}\right] \\
& -\frac{v^{\infty}}{s^{\infty}} S_{\eta}\left[S_{\eta}^{-1}\left\{\frac{1}{\zeta} \frac{\partial}{\partial x} U_{k}(\zeta, s)\right\}\right] .
\end{aligned}
$$

Now, to determine $f_{k}(\zeta), k=1,2,3, \cdots$, we substitute the $k$ th-truncated series (45) into the $k$ th-Shehu residual function (46), multiply the resulting equation by $s^{k ळ+2}$ and, then, solve recursively the relation $\lim _{s \rightarrow \infty}\left[(v / s)^{n \omega+1} \operatorname{Res}_{k}(\zeta, s)\right]=$ $0, k=1,2,3, \cdots$ for $f_{k}(\zeta)$. The first several component of the series $f_{k}(\zeta, \varphi)$

$$
\begin{gathered}
f_{1}(\zeta)=p-4, \\
f_{2}(\zeta)=0, \\
f_{3}(\zeta)=0 .
\end{gathered}
$$

Substituting the value of $f_{n}(\zeta)(n \geq 1)$ in Eq. (45), we obtain as

$$
\begin{gathered}
U(\zeta, s)=\frac{v}{s}\left(1-\zeta^{2}\right)+\frac{v^{\omega}}{s^{\omega}} f_{1}(\zeta)+\frac{v^{\omega+1}}{s^{\omega+1}} f_{2}(\zeta)+\frac{v^{\omega+2}}{s^{\omega+2}} f_{3}(\zeta)+\cdots, \\
U(\zeta, s)=\frac{v}{s}\left(1-\zeta^{2}\right)+\frac{v^{\omega}}{s^{\omega}}(P-4)+\frac{v^{\omega+1}}{s^{\omega+1}}(0)+\frac{v^{\omega+2}}{s^{\omega+2}}(0)+\cdots, \\
U(\zeta, s)=\frac{v}{s}\left(1-\zeta^{2}\right)+\frac{v^{\omega}}{s^{\omega}}(P-4) .
\end{gathered}
$$

Using inverse Shehu transformation to Eq. (49), we get

$$
u(\zeta, \eta)=1-\zeta^{2}+\frac{(P-4) \eta^{\varpi}}{\Gamma(\varrho+2)}
$$

The above equation put $₫=1$, we get

$$
u(\zeta, \eta)=1-\zeta^{2}+(P-4) \eta .
$$



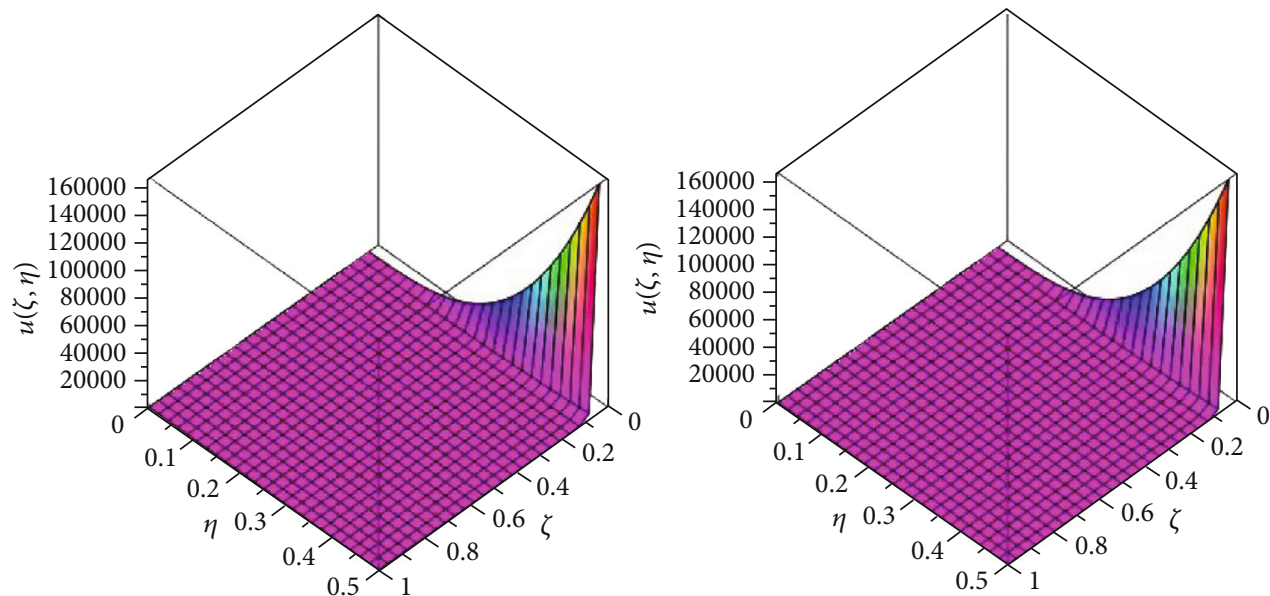

FIGURE 1: Graphs of actual and RPSTM solutions of Example 1.
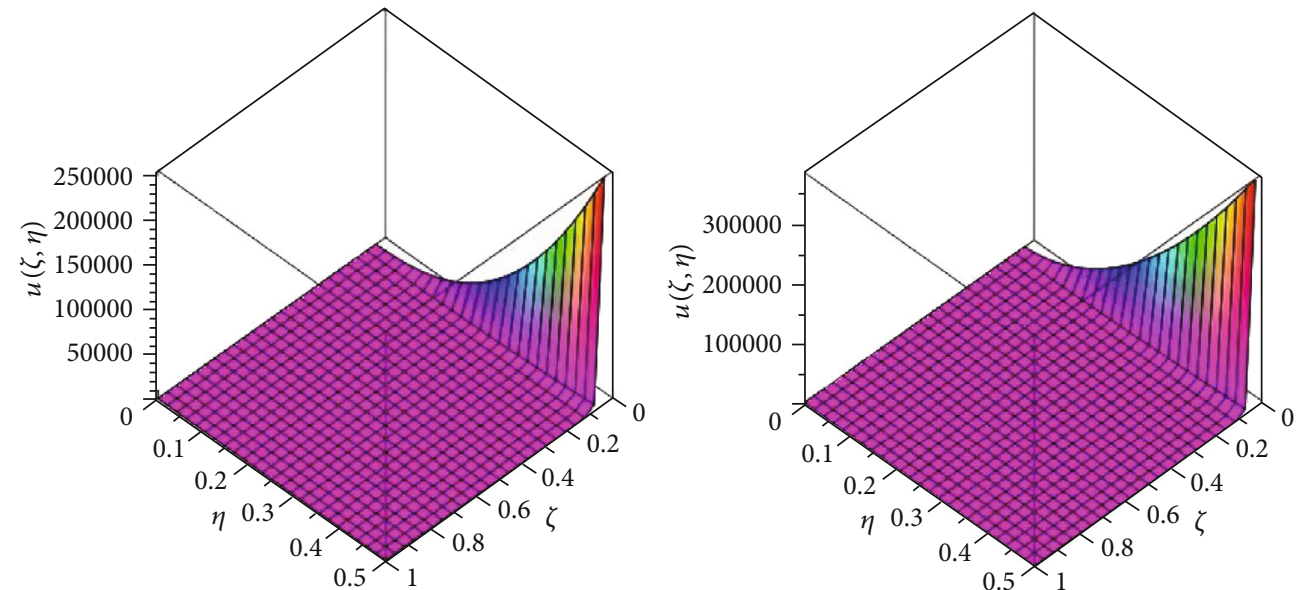

Figure 2: The first graph shows that the fractional-order of $\varpi=0.8$ and the second graph shows that $₫=0.6$ of Example 1 .
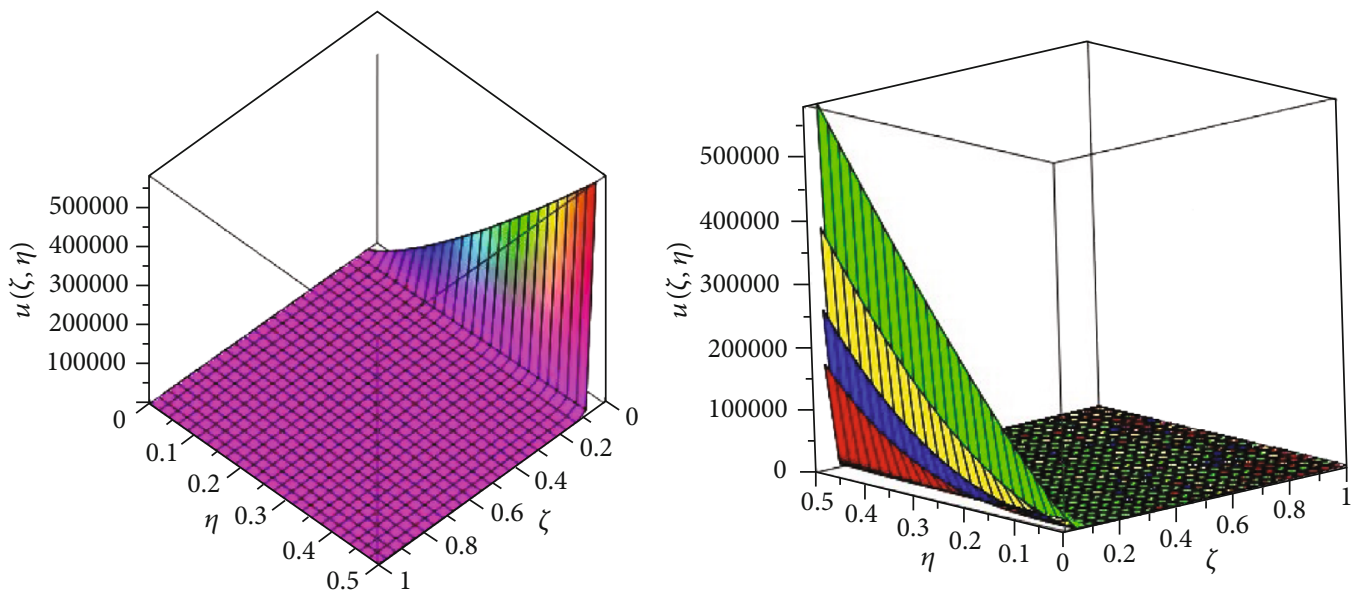

$w=1$

$w=0.6$

$w=0.8$

$w=0.4$

Figure 3: The first graph of fractional-order of $\omega=0.4$ and second graph of the different fractional-order graph of Example 1. 

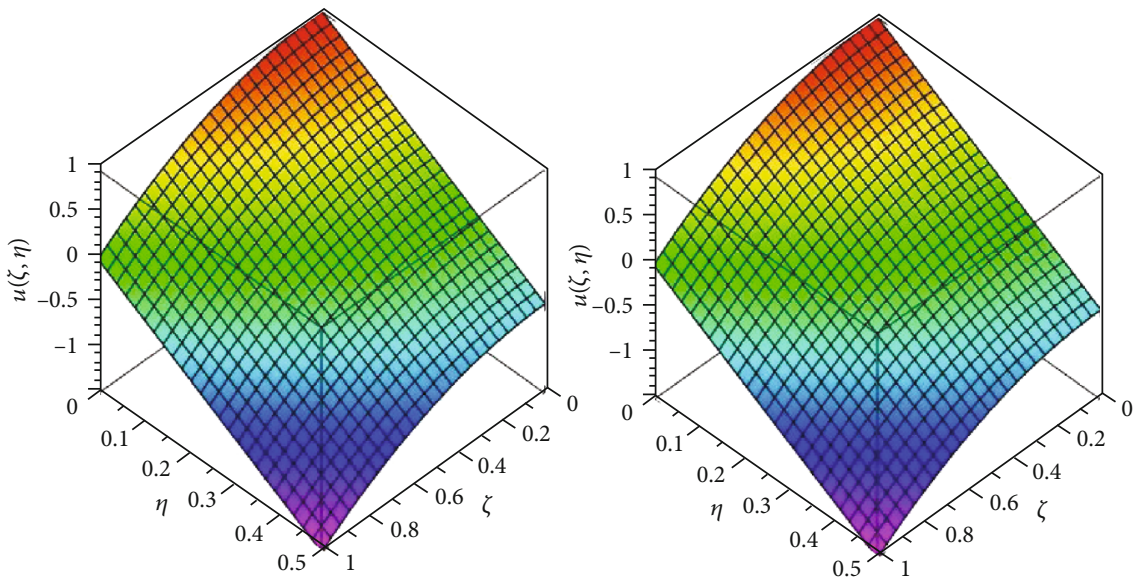

FIGURE 4: Graphs of actual and RPSTM solutions of Example 2.

Figure 4 shows that the actual and analytical solutions graphs at $\square=1$. The field of velocity behavior of the classical Navier-Stokes equations is shown in Figure 4, and the fractional-order behavior of Navier-Stokes equations with $\omega$ $=0.8,0.6$ and 0.4 is shown in Figure 5, respectively.

4.3. Example. Consider the two-dimensional fractional NS equation

$$
\begin{aligned}
& D_{\eta}^{\Phi} u=\rho_{0}\left(\frac{\partial^{2}}{\partial \zeta^{2}} u+\frac{\partial^{2}}{\partial \varphi^{2}} u\right)-u \frac{\partial}{\partial \zeta} u-v \frac{\partial}{\partial \varphi} u+g, \\
& D_{\eta}^{\Phi} v=\rho_{0}\left(\frac{\partial^{2}}{\partial \zeta^{2}} v+\frac{\partial^{2}}{\partial \varphi^{2}} v\right)-u \frac{\partial}{\partial \zeta} v-v \frac{\partial}{\partial \varphi} v-g,
\end{aligned}
$$

with initial condition

$$
u(\zeta, \varphi, 0)=-\sin (\zeta+\varphi), v(\zeta, \varphi, 0)=\sin (\zeta+\varphi)
$$

Applying Shehu transform to Eq. (52) and using the Eq. (53), we get

$$
\begin{aligned}
U(\zeta, \varphi, s)= & \frac{v}{s}(-\sin (\zeta+\varphi))+\rho_{0} \frac{v^{\omega}}{s^{\omega}} S_{\eta} \\
& \cdot\left[S_{\eta}^{-1}\left\{\frac{\partial^{2}}{\partial \zeta^{2}} U_{k}(\zeta, \varphi, s)+\frac{\partial^{2}}{\partial \varphi^{2}} U_{k}(\zeta, \varphi, s)\right\}\right] \\
& -\frac{v^{\omega}}{s^{\omega}} S_{\eta}\left[S_{\eta}^{-1}\left\{U(\zeta, \varphi, s) \frac{\partial}{\partial \zeta} U(\zeta, \varphi, s)\right\}\right] \\
& -\frac{v^{\omega}}{s^{\omega}} S_{\eta}\left[S_{\eta}^{-1}\left\{v(\zeta, \varphi, s) \frac{\partial}{\partial \varphi} U(\zeta, \varphi, s)\right\}\right] \\
& +\frac{v^{\omega}}{s^{\omega}} S_{\eta}[g],
\end{aligned}
$$

$$
\begin{aligned}
V(\zeta, \varphi, s)= & \frac{v}{s}(\sin (\zeta+\varphi))+\rho_{0} \frac{v^{\infty}}{s^{\infty}} S_{\eta} \\
& \cdot\left[S_{\eta}^{-1}\left(\frac{\partial^{2}}{\partial \zeta^{2}} V(\zeta, \varphi, s)+\frac{\partial^{2}}{\partial \varphi^{2}} V(\zeta, \varphi, s)\right)\right] \\
& -\frac{v^{\infty}}{s^{\infty}} S_{\eta}\left[S_{\eta}^{-1}\left\{U(\zeta, \varphi, s) \frac{\partial}{\partial \zeta} V(\zeta, \varphi, s)\right\}\right] \\
& -\frac{v^{\infty}}{s^{\infty}} S_{\eta}\left[S_{\eta}^{-1}\left(V(\zeta, \varphi, s) \frac{\partial}{\partial \varphi} V(\zeta, \varphi, s)\right)\right]-\frac{v^{\infty}}{s^{\omega}} S_{\eta}[g] .
\end{aligned}
$$

The $k$ th truncated term series of Eq. (54) is

$$
\begin{gathered}
U_{k}(\zeta, \varphi, s)=-\sin (\zeta+\varphi) \frac{\nu}{s}+\sum_{n=1}^{k}\left(\frac{\nu}{s}\right)^{n \oplus+1} f_{n}(\zeta, \varphi), \\
V_{k}(\zeta, \varphi, s)=\sin (\zeta+\varphi) \frac{\nu}{s}+\sum_{n=1}^{k}\left(\frac{\nu}{s}\right)^{n \oplus+1} g_{n}(\zeta, \varphi),
\end{gathered}
$$

and the $k$ th Shehu residual function is

$$
\begin{aligned}
& S_{\eta} \operatorname{Res}_{k}(\zeta, \varphi, s)=U_{k}(\zeta, \varphi, s)-(-\sin (\zeta+\varphi)) \frac{v}{s}-\rho_{0} \frac{v^{\omega}}{s^{\omega}} S_{\eta} \\
& \cdot\left[S_{\eta}^{-1}\left\{\frac{\partial^{2}}{\partial \zeta^{2}} U_{k}(\zeta, \varphi, s)+\frac{\partial^{2}}{\partial \varphi^{2}} U_{k}(\zeta, \varphi, s)\right\}\right] \\
& +\frac{v^{\infty}}{s^{\omega}} S_{\eta}\left[S_{\eta}^{-1}\left\{U_{k}(\zeta, \varphi, s) \frac{\partial}{\partial \zeta} U_{k}(\zeta, \varphi, s)\right\}\right] \\
& +\frac{v^{\omega}}{s^{\omega}} S_{\eta}\left[S_{\eta}^{-1}\left\{V_{k}(\zeta, \varphi, s) \frac{\partial}{\partial \varphi} U_{k}(\zeta, \varphi, s)\right\}\right] \\
& -\frac{v^{\omega}}{s^{\omega}} g \text {, }
\end{aligned}
$$




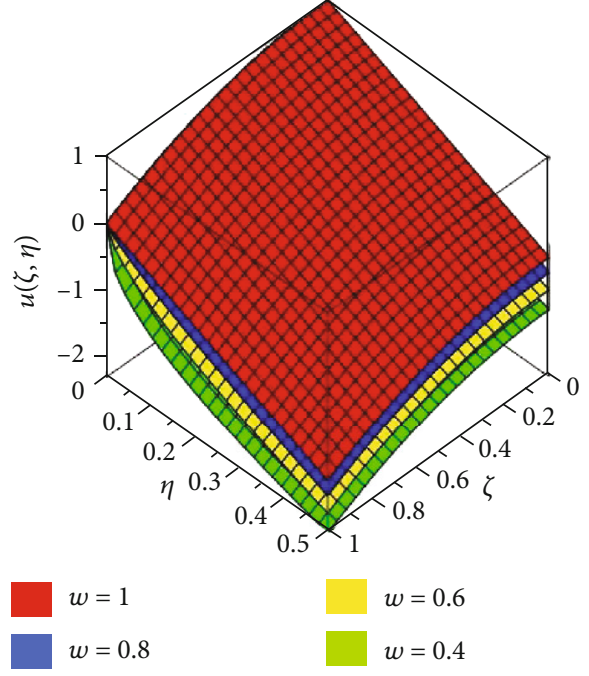

Figure 5: The different fractional-order of $₫$ of Example 2.

$$
\begin{aligned}
S_{\eta} \operatorname{Res}_{k}(\zeta, \varphi, s)= & V_{k}(\zeta, \varphi, s)-\sin (\zeta+\varphi) \frac{v}{s}-\rho_{0} \frac{\nu^{\omega}}{s^{\omega}} S_{\eta} \\
& \cdot\left[S_{\eta}^{-1}\left\{\frac{\partial^{2}}{\partial \zeta^{2}} V_{k}(\zeta, \varphi, s)+\frac{\partial^{2}}{\partial \varphi^{2}} V_{k}(\zeta, \varphi, s)\right\}\right] \\
& +\frac{v^{\omega}}{s^{\omega}} S_{\eta}\left[S_{\eta}^{-1}\left\{U_{k}(\zeta, \varphi, s) \frac{\partial}{\partial \zeta} V_{k}(\zeta, \varphi, s)\right\}\right] \\
& +\frac{v^{\omega}}{s^{\omega}} S_{\eta}\left[S_{\eta}^{-1}\left\{V_{k}(\zeta, \varphi, s) \frac{\partial}{\partial \varphi} V_{k}(\zeta, \varphi, s)\right\}\right] \\
& +\frac{v^{\omega}}{s^{\omega}} g .
\end{aligned}
$$

Now, to determine $f_{k}(\zeta, \varphi)$ and $g_{k}(\zeta, \varphi), k=1,2,3, \cdots$, we substitute the $k$ th-truncated series (55) into the $k$ th-Shehu residual function (56), multiply the resulting equation by $s^{k \omega+2}$, and then solve recursively the relation $\lim _{s \longrightarrow \infty}\left[s^{k \omega+2}\right.$ $\left.\operatorname{Res}_{k}(\zeta, \varphi, s)\right]=0, k=1,2,3, \cdots$ for $f_{k}$ and $g_{k}$. The first few component of the series $f_{k}(\zeta, \varphi)$ and $g_{k}(\zeta, \varphi)$

$$
\begin{gathered}
f_{1}(\zeta, \varphi)=2 \rho_{0} \sin (\zeta+\varphi)+g, \\
g_{1}(\zeta, \varphi)=-2 \rho_{0} \sin (\zeta+\varphi)-g, \\
f_{2}(\zeta, \varphi)=-\left(2 \rho_{0}\right)^{2} \sin (\zeta+\varphi), \\
g_{2}(\zeta, \varphi)=\left(2 \rho_{0}\right)^{2} \sin (\zeta+\varphi), \\
f_{3}(\zeta, \varphi)=\left(2 \rho_{0}\right)^{3} \sin (\zeta+\varphi), \\
g_{3}(\zeta, \varphi)=-\left(2 \rho_{0}\right)^{3} \sin (\zeta+\varphi)
\end{gathered}
$$

Putting the values of $f_{n}(\zeta, \varphi)$ and $g_{n}(\zeta, \varphi),(n \geq 1)$ in Eq.
(55), we get

$$
\begin{aligned}
& U(\zeta, \varphi, s)=-\sin (\zeta+\varphi) \frac{v}{s}+\frac{v^{\omega}}{s^{\omega}} f_{1}(\zeta, \varphi)+\frac{v^{\omega+1}}{s^{\omega+1}} f_{2}(\zeta, \varphi) \\
& +\frac{v^{\oplus+2}}{s^{\oplus+2}} f_{3}(\zeta, \varphi)+\cdots \\
& V(\zeta, \varphi, s)=\sin (\zeta+\varphi) \frac{v}{s}+\frac{v^{\omega}}{s^{\omega}} g_{1}(\zeta, \varphi)+\frac{v^{\omega+1}}{s^{\omega+1}} g_{2}(\zeta, \varphi) \\
& +\frac{v^{\oplus+2}}{s^{\oplus+2}} g_{3}(\zeta, \varphi)+\cdots \\
& U(\zeta, \varphi, s)=-\sin (\zeta+\varphi) \frac{\nu}{s}+\left(2 \rho_{0} \sin (\zeta+\varphi)+g\right) \frac{v^{\omega}}{s^{\omega}} \\
& -\left(2 \rho_{0}\right)^{2} \sin (\zeta+\varphi) \frac{v^{\omega+1}}{s^{\omega+1}}+\left(2 \rho_{0}\right)^{3} \sin (\zeta+\varphi) \frac{v^{\omega+2}}{s^{\omega+2}}+\cdots, \\
& V(\zeta, \varphi, s)=\sin (\zeta+\varphi) \frac{\nu}{s}-\left(2 \rho_{0} \sin (\zeta+\varphi)-g\right) \frac{v^{\omega}}{s^{\omega}} \\
& +\left(2 \rho_{0}\right)^{2} \sin (\zeta+\varphi) \frac{v^{\omega+1}}{s^{\omega+1}}-\left(2 \rho_{0}\right)^{3} \sin (\zeta+\varphi) \frac{v^{\omega+2}}{s^{\omega+2}}+\cdots, \\
& U(\zeta, \varphi, s)=-\sin (\zeta+\varphi)\left[\frac{\nu}{s}-2 \rho_{0} \frac{v^{\omega}}{s^{\omega}}+\left(2 \rho_{0}\right)^{2} \frac{v^{\omega+1}}{s^{\omega+1}}-\left(2 \rho_{0}\right)^{3} \frac{v^{\omega+2}}{s^{\omega+2}}+\cdots\right] \\
& +g \frac{v^{\omega}}{s^{\omega}}, \\
& V(\zeta, \varphi, s)=\sin (\zeta+\varphi)\left[\frac{\nu}{s}-2 \rho_{0} \frac{v^{\omega}}{s^{\omega}}+\left(2 \rho_{0}\right)^{2} \frac{v^{\omega+1}}{s^{\omega+1}}-\left(2 \rho_{0}\right)^{3} \frac{v^{\omega+2}}{s^{\omega+2}}+\cdots\right] \\
& -g \frac{v^{\omega}}{s^{\omega}} .
\end{aligned}
$$

Using inverse Shehu transformation, we get

$$
\begin{aligned}
& u(\zeta, \varphi, \eta)=-\sin (\zeta+\varphi)\left[1-\frac{2 \rho_{0} \eta^{\varpi}}{\Gamma(\omega+2)}+\frac{\left(2 \rho_{0}\right)^{2} \eta^{2 \omega}}{\Gamma(2 \omega+2)}-\frac{\left(2 \rho_{0}\right)^{3} \eta^{3 \omega}}{\Gamma(3 \omega+2)}+\cdots\right] \\
& +g \frac{\eta^{\varpi}}{\Gamma(\omega+2)}, \\
& \begin{aligned}
v(\zeta, \varphi, \eta)= & \sin (\zeta+\varphi)\left[1-\frac{2 \rho_{0} \eta^{\Phi}}{\Gamma(\omega+2)}+\frac{\left(2 \rho_{0}\right)^{2} \eta^{2 ळ}}{\Gamma(2 \omega+2)}-\frac{\left(2 \rho_{0}\right)^{3} \eta^{3 ळ}}{\Gamma(3 \omega+2)}+\cdots\right] \\
& -g \frac{\eta^{\Phi}}{\Gamma(\omega+2)}
\end{aligned}
\end{aligned}
$$

The exact solution is given as

$$
\begin{gathered}
u(\zeta, \varphi, \eta)=-\sin (\zeta+\varphi) e^{-2 \rho_{0} \eta}+g \\
v(\zeta, \varphi, \eta)=\sin (\zeta+\varphi) e^{-2 \rho_{0} \eta}-g .
\end{gathered}
$$

Figure 6 shows that the actual and analytical solution graphs at $\omega=1$ with respect to $u(\zeta, \varphi, \eta)$. The field of velocity behavior of the classical Navier-Stokes equations is shown in Figure 6, and the fractional-order behavior of Navier-Stokes equations with $\omega=0.8$ and 0.6 is shown in Figure 7 , 

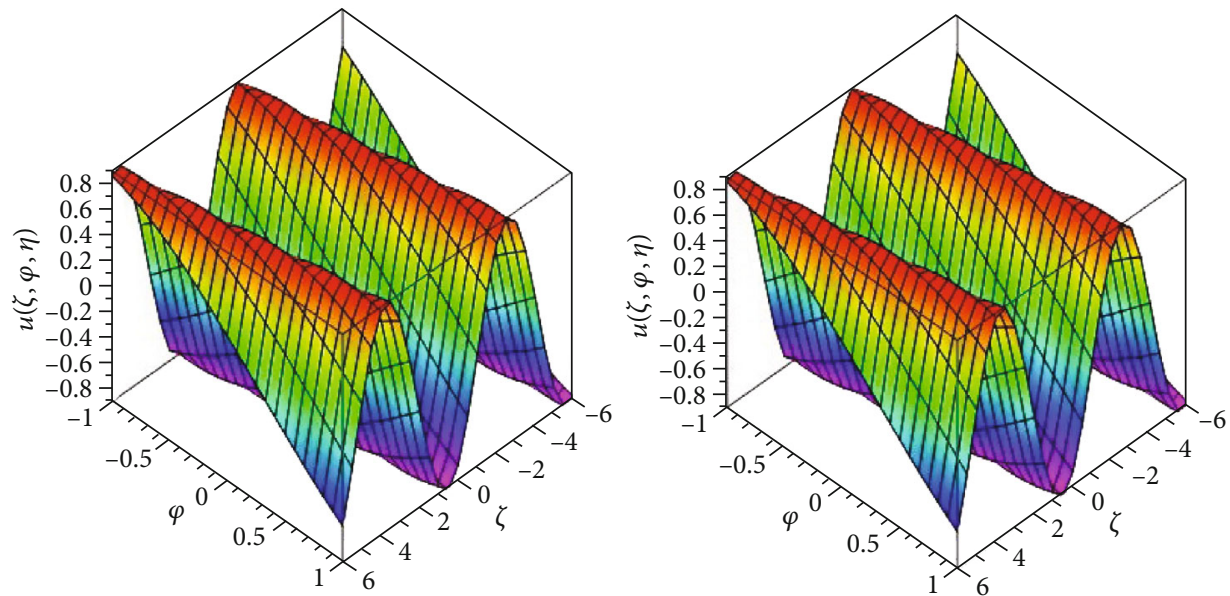

FIgURE 6: Graphs of actual and approximate solutions of Example 3.
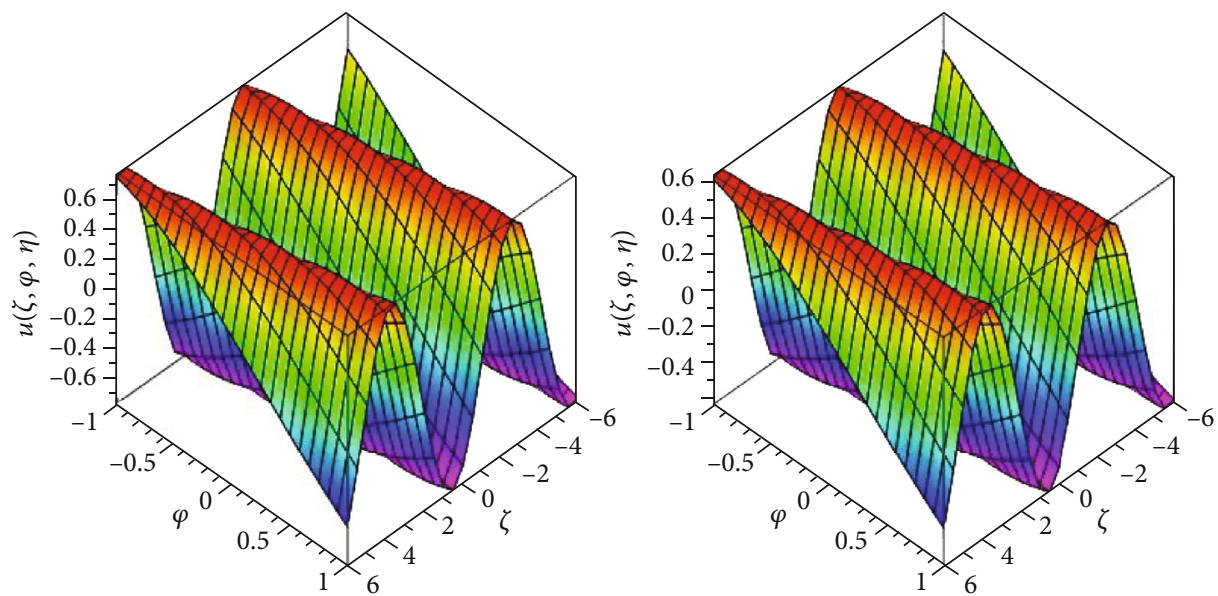

Figure 7: The first graph shows that the fractional-order of $₫=0.8$ and the second graph shows that the fractional-order $₫=0.6$ of Example 3.
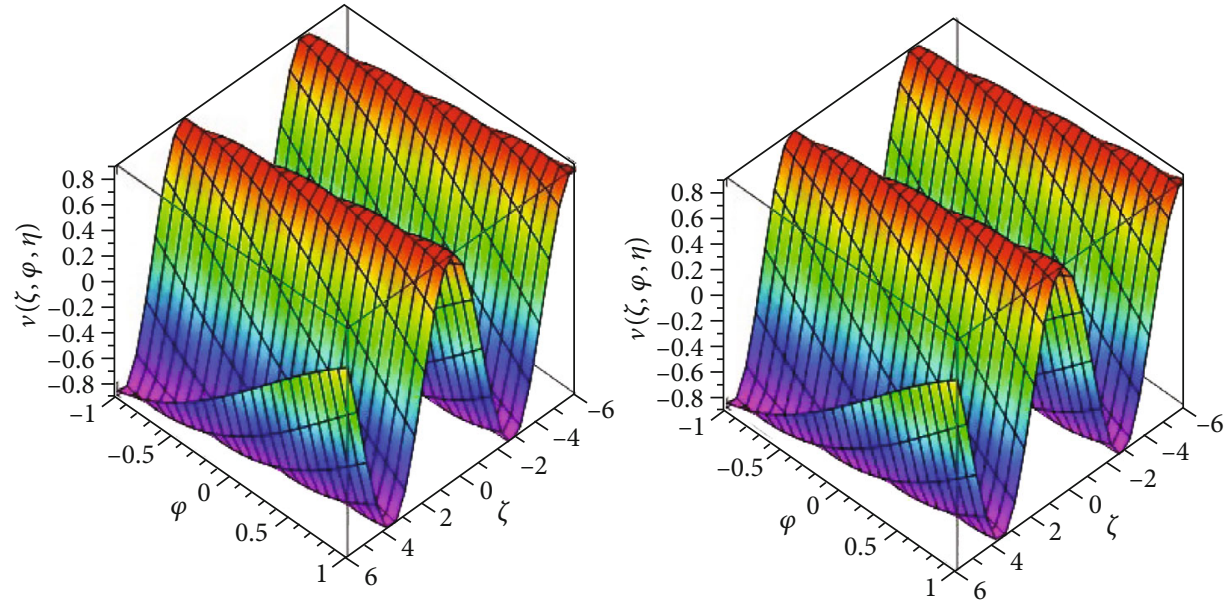

Figure 8: Graphs of actual and approximate solutions of Example 3. 

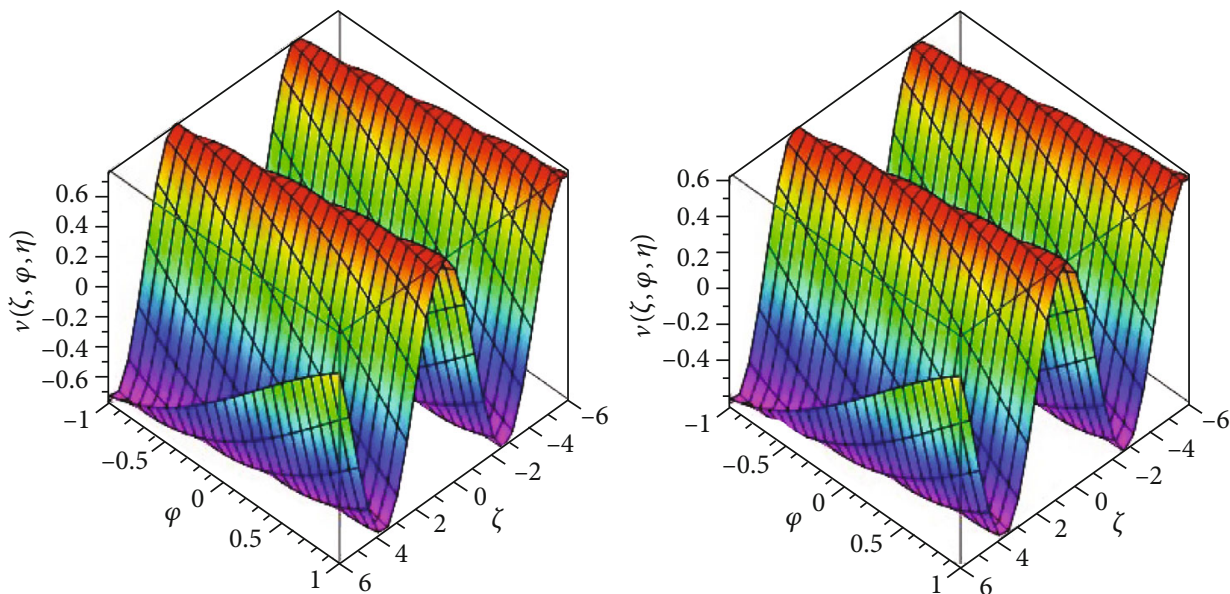

Figure 9: The first graph shows that the fractional-order of $₫=0.8$ and the second graph shows that the fractional-order $₫=0.6$ of Example 3.

respectively. Similarly, Figure 8 shows that the actual and analytical solution graphs at $\Phi=1$ with respect to $v(\zeta, \varphi, \eta)$. The field of velocity behavior of the classical Navier-Stokes equations is shown in Figure 8, and the fractional-order behavior of Navier-Stokes equations with $₫=0.8$ and 0.6 is shown in Figure 9, respectively.

4.4. Example. Consider the two-dimensional fractional NS equation

$$
\begin{aligned}
& D_{\eta}^{\Phi} u=\rho_{0}\left(\frac{\partial^{2}}{\partial \zeta^{2}} u+\frac{\partial^{2}}{\partial \varphi^{2}} u\right)-u \frac{\partial}{\partial \zeta} u-v \frac{\partial}{\partial \varphi} u+g, \\
& D_{\eta}^{\Phi} v=\rho_{0}\left(\frac{\partial^{2}}{\partial \zeta^{2}} v+\frac{\partial^{2}}{\partial \varphi^{2}} v\right)-u \frac{\partial}{\partial \zeta} v-v \frac{\partial}{\partial \varphi} v-g,
\end{aligned}
$$

with initial condition

$$
u(\zeta, \varphi, 0)=-e^{\zeta+\varphi}, v(\zeta, \varphi, 0)=e^{\zeta+\varphi} .
$$

Applying Shehu transform to Eq. (61) and using the Eq. (62), we obtain as

$$
\begin{aligned}
& U(\zeta, \varphi, s)=-e^{\zeta+\varphi} \frac{\nu}{s}+\rho_{0} \frac{v^{\Phi}}{s^{\omega}} S_{\eta}\left[S_{\eta}^{-1}\left\{\frac{\partial^{2}}{\partial \zeta^{2}} U_{k}(\zeta, \varphi, s)+\partial^{2} \partial \varphi^{2} U_{k}(\zeta, \varphi, s)\right\}\right] \\
& -\frac{v^{\omega}}{s^{\omega}} S_{\eta}\left[S_{\eta}^{-1}\left\{U(\zeta, \varphi, s) \frac{\partial}{\partial \zeta} U(\zeta, \varphi, s)\right\}\right]-\frac{v^{\omega}}{s^{\omega}} S_{\eta} \\
& \cdot\left[S_{\eta}^{-1}\left\{v(\zeta, \varphi, s) \frac{\partial}{\partial \varphi} U(\zeta, \varphi, s)\right\}\right]+\frac{v^{\omega}}{s^{\omega}} S_{\eta}[g], \\
& V(\zeta, \varphi, s)=e^{\zeta+\varphi} \frac{\nu}{s}+\rho_{0} \frac{v^{\infty}}{s^{\infty}} S_{\eta}\left[S_{\eta}^{-1}\left\{\frac{\partial^{2}}{\partial \zeta^{2}} V(\zeta, \varphi, s)+\frac{\partial^{2}}{\partial \varphi^{2}} V(\zeta, \varphi, s)\right\}\right] \\
& -\frac{v^{\infty}}{s^{\omega}} S_{\eta}\left[S_{\eta}^{-1}\left\{U(\zeta, \varphi, s) \frac{\partial}{\partial \zeta} V(\zeta, \varphi, s)\right\}\right] \\
& -\frac{v^{\Phi}}{s^{\infty}} S_{\eta}\left[S_{\eta}^{-1}\left\{V(\zeta, \varphi, s) \frac{\partial}{\partial \varphi} V(\zeta, \varphi, s)\right\}\right]-\frac{v^{\Phi}}{s^{\omega}} S_{\eta}[g] .
\end{aligned}
$$

The $k$ th truncated term series of Eq. (63) is

$$
\begin{aligned}
& U_{k}(\zeta, \varphi, s)=-e^{\zeta+\varphi} \frac{\nu}{s}+\sum_{n=1}^{k}\left(\frac{\nu}{s}\right)^{n \omega+1} f_{n}(\zeta, \varphi), \\
& V_{k}(\zeta, \varphi, s)=e^{\zeta+\varphi} \frac{\nu}{s}+\sum_{n=1}^{k}\left(\frac{\nu}{s}\right)^{n \omega+1} g_{n}(\zeta, \varphi),
\end{aligned}
$$

and the $k$ th Shehu residual function is

$$
\begin{aligned}
S_{\eta} \operatorname{Res}_{k}(\zeta, \varphi, s)= & U_{k}(\zeta, \varphi, s)-e^{\zeta+\varphi} \frac{\nu}{s}-\rho_{0} \frac{v^{\Phi}}{s^{\omega}} S_{\eta} \\
& \cdot\left[S_{\eta}^{-1}\left\{\frac{\partial^{2}}{\partial \zeta^{2}} U_{k}(\zeta, \varphi, s)+\frac{\partial^{2}}{\partial \varphi^{2}} U_{k}(\zeta, \varphi, s)\right\}\right] \\
& +\frac{v^{\infty}}{s^{\omega}} S_{\eta}\left[S_{\eta}^{-1}\left\{U_{k}(\zeta, \varphi, s) \frac{\partial}{\partial \zeta} U_{k}(\zeta, \varphi, s)\right\}\right] \\
& +\frac{v^{\infty}}{s^{\omega}} S_{\eta}\left[S_{\eta}^{-1}\left\{V_{k}(\zeta, \varphi, s) \frac{\partial}{\partial \varphi} U_{k}(\zeta, \varphi, s)\right\}\right] \\
& -g \frac{1}{v^{\omega} / s^{\omega}}, \\
S_{\eta} \operatorname{Res}_{k}(\zeta, \varphi, s)= & V_{k}(\zeta, \varphi, s)-e^{\zeta+\varphi} \frac{\nu}{s}-\rho_{0} \frac{v^{\infty}}{s^{\omega}} S_{\eta} \\
& +\left[S_{\eta}^{-1}\left\{\frac{\partial^{2}}{\partial \zeta^{2}} V_{k}(\zeta, \varphi, s)+\frac{\partial^{2}}{\partial \varphi^{2}} V_{k}(\zeta, \varphi, s)\right\}\right] \\
& +\frac{v^{\infty}}{s^{\omega}} S_{\eta}\left[S_{\eta}^{-1}\left\{U_{k}(\zeta, \varphi, s) \frac{\partial}{\partial \zeta} V_{k}(\zeta, \varphi, s)\right\}\right] \\
& +\frac{v^{\infty}}{s^{\omega}} S_{\eta}\left[S_{\eta}^{-1}\left\{V_{k}(\zeta, \varphi, s) \frac{\partial}{\partial \varphi} V_{k}(\zeta, \varphi, s)\right\}\right] \\
& +g \frac{1}{v^{\omega} / s^{\omega}} .
\end{aligned}
$$

Now, to determine $f_{k}(\zeta, \varphi)$ and $g_{k}(\zeta, \varphi), k=1,2,3, \cdots$, 

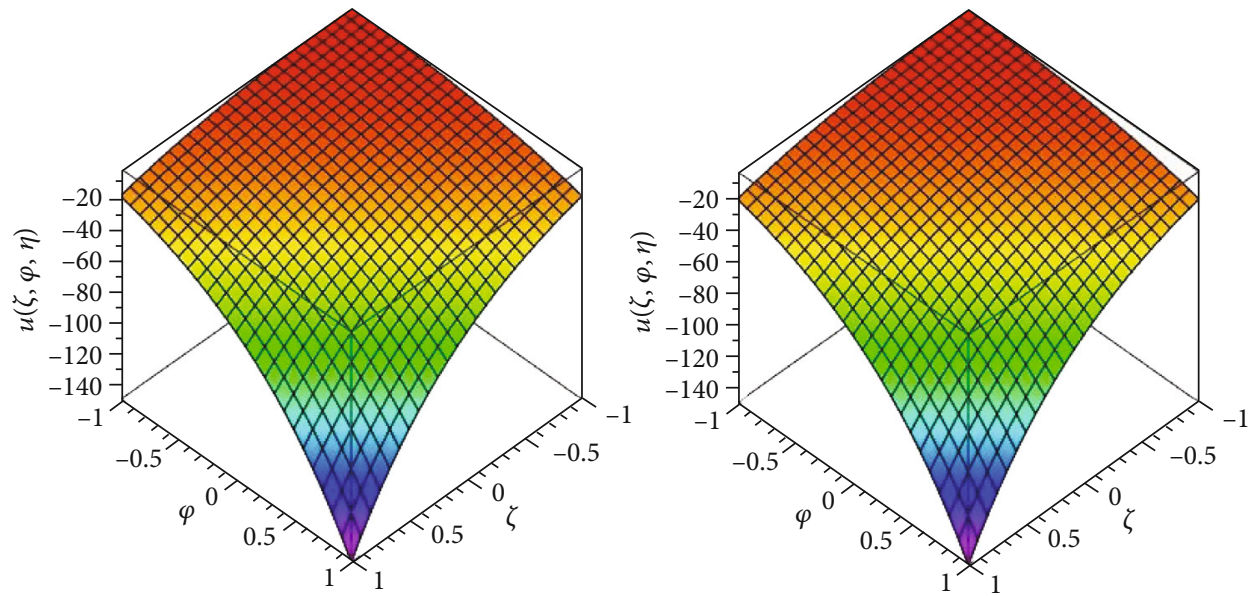

FIgURE 10: Graphs of actual and approximate solutions of Problem 4.
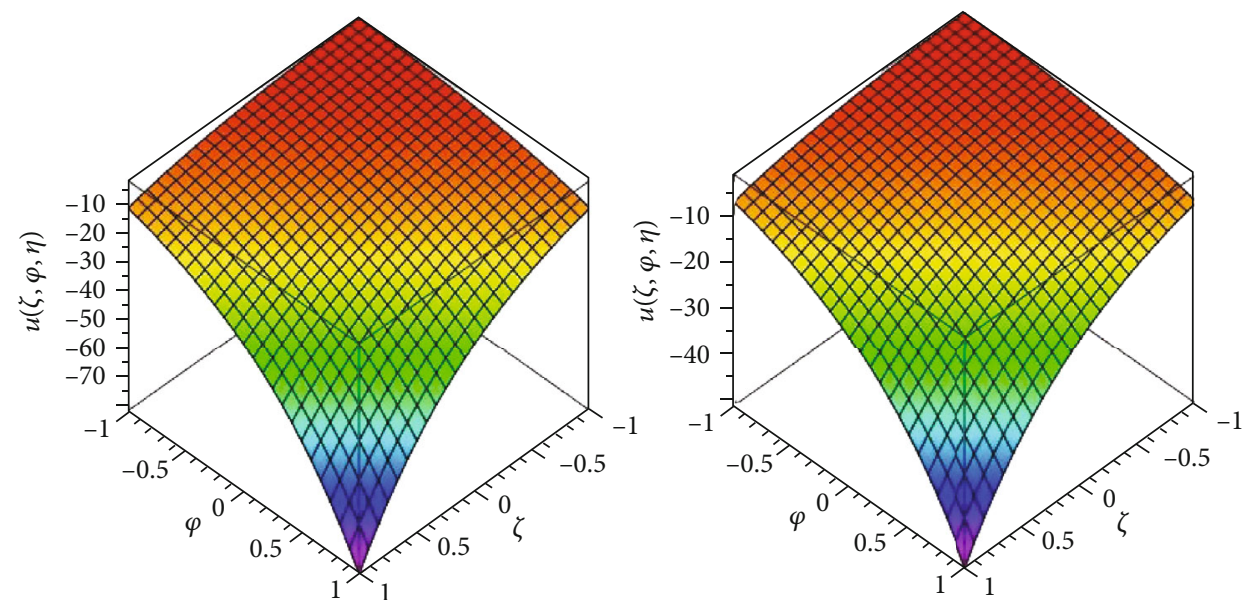

FIGURE 11: The first graph shows that the fractional-order of $\omega=0.8$ and the second graph shows that the fractional-order $\emptyset=0.6$ of Example 4.

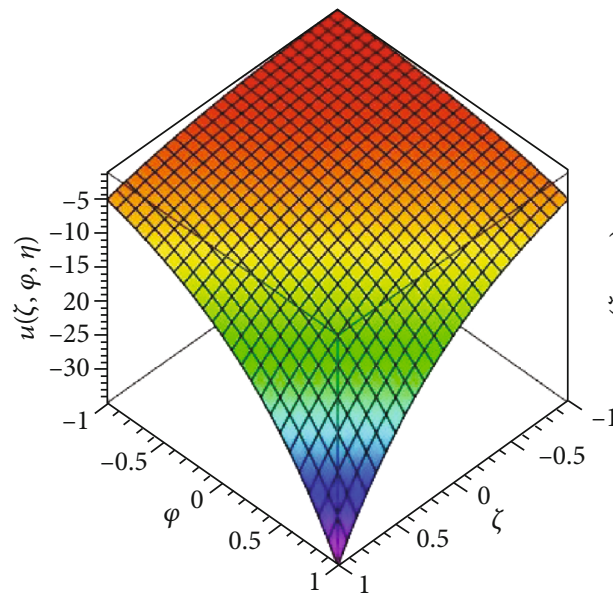

$w=1$

$w=0.8$

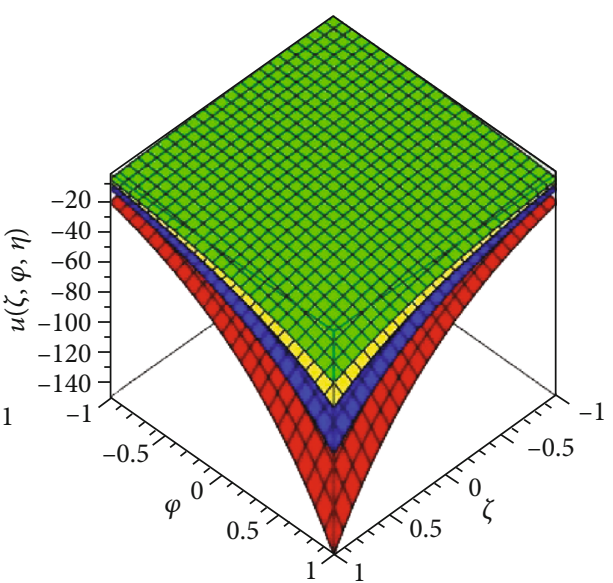

$w=0.6$

$w=0.4$

Figure 12: The first graph of fractional-order of $\omega=0.4$ and second graph of the different fractional-order graph of Example 4. 

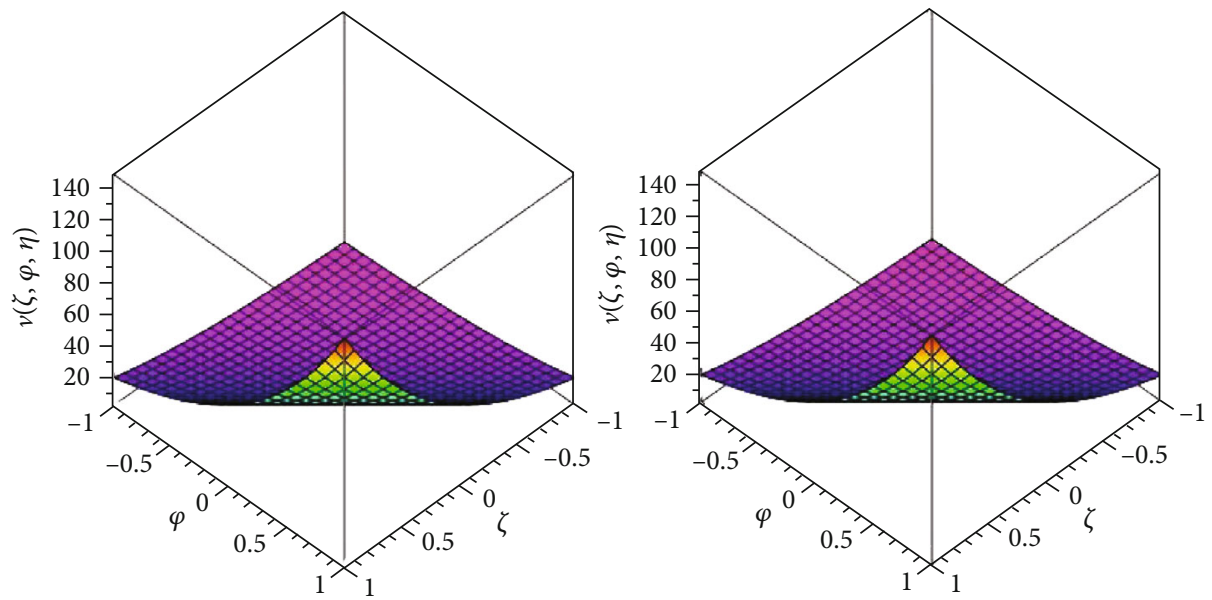

FIGURE 13: Graphs of actual and approximate solutions of Example 4.
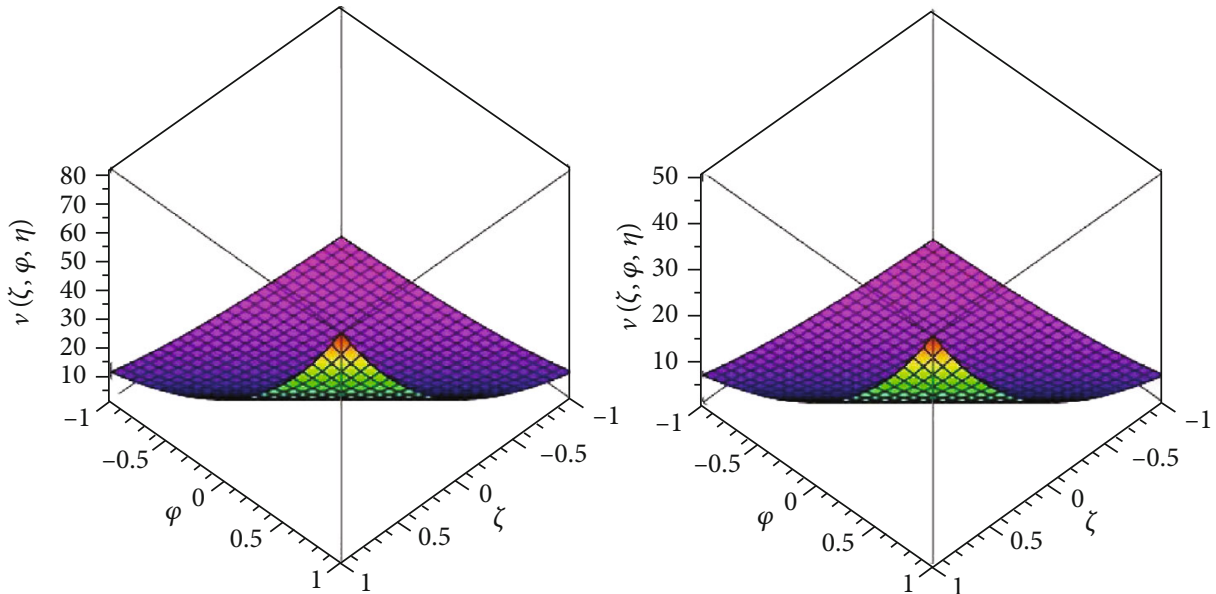

FIgURE 14: The first graph shows that the fractional-order of $\omega=0.8$ and the second graph shows that the fractional-order $\omega=0.6$ of Example 4.
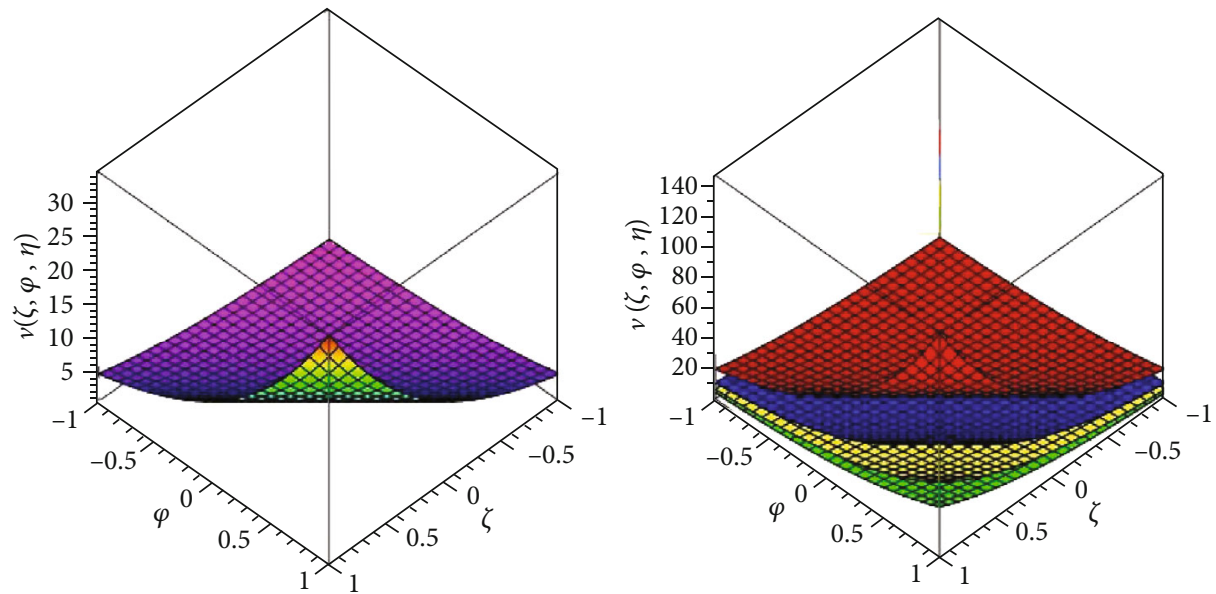

$w=1$

$w=0.6$

$w=0.8$

$w=0.4$

FIgURE 15: The first graph of fractional-order of $₫=0.4$ and second graph of the different fractional-order graph of Example 4. 
we substitute the $k$ th-truncated series (64) into the $k$ th-Shehu residual function (65), multiply the resulting equation by $s^{k \omega+2}$, and then solve recursively the relation $\lim _{s \longrightarrow \infty}[$ $\left.(\nu / s)^{n \omega+1} \operatorname{Res}_{k}(\zeta, \varphi, s)\right]=0, k=1,2,3, \cdots$ for $f_{k}$ and $g_{k}$. The first few component of the series $f_{k}(\zeta, \varphi)$ and $g_{k}(\zeta, \varphi)$

$$
\begin{gathered}
f_{1}(\zeta, \varphi)=-2 \rho_{0} e^{\zeta+\varphi}+g \\
g_{1}(\zeta, \varphi)=2 \rho_{0} e^{\zeta+\varphi}-g, \\
f_{2}(\zeta, \varphi)=-\left(2 \rho_{0}\right)^{2} e^{\zeta+\varphi}, \\
g_{2}(\zeta, \varphi)=\left(2 \rho_{0}\right)^{2} e^{\zeta+\varphi}, \\
f_{3}(\zeta, \varphi)=-\left(2 \rho_{0}\right)^{3} e^{\zeta+\varphi}, \\
g_{3}(\zeta, \varphi)=\left(2 \rho_{0}\right)^{3} e^{\zeta+\varphi} .
\end{gathered}
$$

Putting the values of $f_{n}(\zeta, \varphi)$ and $g_{n}(\zeta, \varphi),(n \geq 1)$ in Eq. (64), we get

$$
\begin{aligned}
& U(\zeta, \varphi, s)=-e^{\zeta+\varphi} \frac{v}{s}+f_{1}(\zeta, \varphi) \frac{v^{\omega}}{s^{\omega}}+f_{2}(\zeta, \varphi) \frac{v^{\omega+1}}{s^{\omega+1}}+f_{3}(\zeta, \varphi) \frac{v^{\omega+2}}{s^{\omega+2}}+\cdots \\
& V(\zeta, \varphi, s)=e^{\zeta+\varphi} \frac{v}{s}+g_{1}(\zeta, \varphi) \frac{v^{\omega}}{s^{\omega}}+g_{2}(\zeta, \varphi) \frac{v^{\omega+1}}{s^{\omega+1}}+g_{3}(\zeta, \varphi) \frac{v^{\omega+2}}{s^{\omega+2}}+\cdots, \\
& U(\zeta, \varphi, s)=-e^{\zeta+\varphi} \frac{\nu}{s}-2 \rho_{0} e^{\zeta+\varphi}+g \frac{v^{\omega}}{s^{\omega}}-\left(2 \rho_{0}\right)^{2} e^{\zeta+\varphi} \frac{v^{\omega+1}}{s^{\omega+1}}-\left(2 \rho_{0}\right)^{3} e^{\zeta+\varphi} \frac{v^{\omega+2}}{s^{\omega+2}}+\cdots, \\
& V(\zeta, \varphi, s)=e^{\zeta+\varphi} \frac{\nu}{s}+2 \rho_{0} e^{\zeta+\varphi}-g \frac{v^{\omega}}{s^{\omega}}+\left(2 \rho_{0}\right)^{2} e^{\zeta+\varphi} \frac{v^{\omega+1}}{s^{\omega+1}}+\left(2 \rho_{0}\right)^{3} e^{\zeta+\varphi} \frac{v^{\omega+2}}{s^{\omega+2}}+\cdots, \\
& U(\zeta, \varphi, s)=-e^{\zeta+\varphi}\left[\frac{v}{s}+2 \rho_{0} \frac{v^{\omega}}{s^{\omega}}+\left(2 \rho_{0}\right)^{2} \frac{v^{\omega+1}}{s^{\omega+1}}+\left(2 \rho_{0}\right)^{3} \frac{v^{\omega+2}}{s^{\omega+2}}+\cdots\right]+g \frac{v^{\omega}}{s^{\omega}}, \\
& V(\zeta, \varphi, s)=e^{\zeta+\varphi}\left[\frac{\nu}{s}+2 \rho_{0} \frac{v^{\omega}}{s^{\omega}}+\left(2 \rho_{0}\right)^{2} \frac{v^{\omega+1}}{s^{\omega+1}}+\left(2 \rho_{0}\right)^{3} \frac{v^{\omega+2}}{s^{\omega+2}}+\cdots\right]-g \frac{v^{\omega}}{s^{\omega}} .
\end{aligned}
$$

Using inverse Shehu transformation, we get

$$
\begin{aligned}
& u(\zeta, \varphi, \eta)=-e^{\zeta+\varphi}\left[1+\frac{2 \rho_{0} \eta^{\omega}}{\Gamma(\omega+2)}+\frac{\left(2 \rho_{0}\right)^{2} \eta^{2 \omega}}{\Gamma(2 \omega+2)}+\frac{\left(2 \rho_{0}\right)^{3} \eta^{3 \omega}}{\Gamma(3 \omega+2)}+\cdots\right]+g \frac{\eta^{\omega}}{\Gamma(\omega+2)}, \\
& v(\zeta, \varphi, \eta)=e^{\zeta+\varphi}\left[1+\frac{2 \rho_{0} \eta^{\Phi}}{\Gamma(\omega+2)}+\frac{\left(2 \rho_{0}\right)^{2} \eta^{2 \omega}}{\Gamma(2 \omega+2)}+\frac{\left(2 \rho_{0}\right)^{3} \eta^{3 \omega}}{\Gamma(3 \omega+2)}+\cdots\right]-g \frac{\eta^{\omega}}{\Gamma(\varpi+2)} .
\end{aligned}
$$

The exact result is given as

$$
\begin{aligned}
& u(\zeta, \varphi, \eta)=-e^{\zeta+\varphi+2 \rho_{0} \eta}+g \\
& v(\zeta, \varphi, \eta)=e^{\zeta+\varphi+2 \rho_{0} \eta}-g
\end{aligned}
$$

Figure 10 shows that the actual and analytical solution graphs at $\Phi=1$ with respect to $u(\zeta, \varphi, \eta)$. The field of velocity behavior of the classical Navier-Stokes equations is shown in Figure 10, and the fractional-order behavior of Navier-Stokes equations with $\omega=0.8,0.6$ and 0.4 is shown in Figures 11 and 12, respectively. Similarly, Figure 13 shows that the actual and analytical solution graphs at $\omega=1$ with respect to $v(\zeta$, $\varphi, \eta)$. The field of velocity behavior of the classical NavierStokes equations is show in Figure 13, and the fractionalorder behavior of Navier-Stokes equations with $\omega=0.8$ and 0.6 is shown in Figures 14 and 15, respectively.

\section{Conclusion}

In this paper, the residual power series transform method is implemented to approximate analytical solutions of the fractional-order Navier-Stokes equations, which are widely applied as problems for spatial effects in applied sciences. The method gives a series form of solutions that converge very quickly in physical problems. It is predicted that this paper obtains solutions to analyze the complicated nonlinear physical problems. The calculations of this method are very simple and straightforward. Thus, we assume that this method can be applied to solve many systems of nonlinear fractional-order partial differential equations.

\section{Data Availability}

The numerical data used to support the findings of this study are included within the article.

\section{Conflicts of Interest}

The authors declare that there are no conflicts of interest regarding the publication of this article.

\section{Acknowledgments}

One of the coauthors (A. M. Zidan) extends his appreciation to the Deanship of Scientific Research at King Khalid University, Abha 61413, Saudi Arabia, for funding this work through research groups program under grant number R.G.P-2/142/42.

\section{References}

[1] L. Xu, Y.-M. Chu, S. Rashid, A. A. El-Deeb, and K. S. Nisar, "On new unified bounds for a family of functions via fractional-calculus theory," Journal of Function Spaces, vol. 2020, Article ID 4984612, 9 pages, 2020.

[2] N. I. Mahmudov and M. A. McKibben, "On the approximate controllability of fractional evolution equations with generalized Riemann-Liouville fractional derivative," Journal of Function Spaces, vol. 2015, Article ID 263823, 9 pages, 2015.

[3] R. Gorenflo and F. Mainardi, "Fractional calculus," in Fractals and fractional calculus in continuum mechanics, pp. 223-276, Springer, Vienna, Austria, 1997.

[4] C. Guendouz, J. E. Lazreg, J. J. Nieto, and A. Ouahab, "Existence and compactness results for a system of fractional differential equations," Journal of Function Spaces, vol. 2020, Article ID 5735140, 12 pages, 2020.

[5] J. A. Sabatier, O. P. Agrawal, and J. T. Machado, Advances in fractional calculus, Springer, Dordrecht, Netherlands, 2007.

[6] J.-H. He, "Max-min approach to nonlinear oscillators," Moon, vol. 1 , no. 29, p. 399, 2008. 
[7] J.-H. He, "Homotopy perturbation technique," Computer Methods in Applied Mechanics and Engineering, vol. 178, no. 3-4, pp. 257-262, 1999.

[8] J.-H. He, "Approximate analytical solution for seepage flow with fractional derivatives in porous media," Computer Methods in Applied Mechanics and Engineering, vol. 167, no. 1-2, pp. 57-68, 1998.

[9] M. Caputo, "Elasticita de dissipazione, Zanichelli, Bologna, Italy,(Links)," SIAM Journal on Numerical Analysis, 1969.

[10] K. B. Oldham and J. Spanier, The Fractional Calculus Theory and Applications of Differentiation and Integration to Arbitrary Order, Academic Press, New York, NY, USA, 1974.

[11] K. S. Miller and B. Ross, An Introduction to the Fractional Calculus and Fractional Differential Equations, Wiley, New York, NY, USA, 1993.

[12] G. O. Young, "Definition of physical consistent damping laws with fractional derivatives," Zeitschrift für Angewandte Mathematik und Mechanik, vol. 75, pp. 623-635, 1995.

[13] I. Podlubny, Fractional Differential Equations, Academic Press, New York, 1999.

[14] R. Hilfer, Ed., Applications of Fractional Calculus in Physics, World Scientific Publishing Company, 2000.

[15] F. Mainardi, Y. Luchko, and G. Pagnini, "The fundamental solution of the space-time fractional diffusion equation," Fractional Calculus and Applied Analysis, vol. 4, pp. 153192, 2001.

[16] L. Debnath, "Fractional integrals and fractional differential equations in fluid mechanics," Fractional Calculus and Applied Analysis, vol. 6, pp. 119-155, 2003.

[17] A. A. Kilbas, H. M. Srivastava, and J. J. Trujillo, Theory and Applications of Fractional Differential Equations, Elsevier, Amsterdam, Netherlands, 2006.

[18] C. L. M. H. Navier, "Mémoire sur les lois du mouvement des fluides," Mémoires de l'Académie Royale des Sciences de l'Institut de France, vol. 6, no. 1823, pp. 389-440, 1823.

[19] M. El-Shahed and A. Salem, "On the generalized NavierStokes equations," Applied Mathematics and Computation, vol. 156, no. 1, pp. 287-293, 2004.

[20] S. Momani and Z. Odibat, "Analytical solution of a timefractional Navier-Stokes equation by Adomian decomposition method," Applied Mathematics and Computation, vol. 177, no. 2, pp. 488-494, 2006.

[21] Z. Z. Ganji, D. D. Ganji, A. D. Ganji, and M. Rostamian, “Analytical solution of time-fractional Navier-Stokes equation in polar coordinate by homotopy perturbation method," Numerical Methods for Partial Differential Equations, vol. 26, no. 1, pp. 117-124, 2010.

[22] B. K. Singh and P. Kumar, "FRDTM for numerical simulation of multi-dimensional, time-fractional model of Navier-Stokes equation," Ain Shams Engineering Journal, vol. 9, no. 4, pp. 827-834, 2018.

[23] K. K. Jaber and R. S. Ahmad, "Analytical solution of the time fractional Navier-stokes equation," Ain Shams Engineering Journal, vol. 9, no. 4, pp. 1917-1927, 2018.

[24] J. Zhang and J. Wang, "Numerical analysis for Navier-Stokes equations with time fractional derivatives," Applied Mathematics and Computation, vol. 336, pp. 481-489, 2018.

[25] D. Kumar, J. Singh, and S. Kumar, "A fractional model of Navier-Stokes equation arising in unsteady flow of a viscous fluid," Journal of the Association of Arab Universities for Basic and Applied Sciences, vol. 17, no. 1, pp. 14-19, 2015.

[26] R. M. Jena and S. Chakraverty, "Solving time-fractional Navier-Stokes equations using homotopy perturbation Elzaki transform," SN Applied Sciences, vol. 1, no. 1, p. 16, 2019.

[27] A. Prakash, P. Veeresha, D. G. Prakasha, and M. Goyal, "A new efficient technique for solving fractional coupled NavierStokes equations using q-homotopy analysis transform method," Pramana, vol. 93, no. 1, p. 6, 2019.

[28] M. Alquran, M. Ali, M. Alsukhour, and I. Jaradat, "Promoted residual power series technique with Laplace transform to solve some time-fractional problems arising in physics," Results in Physics, vol. 19, article 103667, 2020.

[29] O. A. Arqub, "Series solution of fuzzy differential equations under strongly generalized differentiability," Journal of Advanced Research in Applied Mathematics, vol. 5, no. 1, pp. 31-52, 2013.

[30] O. Abu Arqub, A. El-Ajou, A. S. Bataineh, and I. Hashim, "A representation of the exact solution of generalized LaneEmden equations using a new analytical method," Abstract and Applied Analysis, vol. 2013, Article ID 378593, 10 pages, 2013.

[31] O. Abu Arqub, Z. Abo-Hammour, R. Al-Badarneh, and S. Momani, "A reliable analytical method for solving higherorder initial value problems," Discrete Dynamics in Nature and Society, vol. 2013, 12 pages, 2013.

[32] A. el-Ajou, O. A. Arqub, Z. A. Zhour, and S. Momani, "New results on fractional power series: theories and applications," Entropy, vol. 15, no. 12, pp. 5305-5323, 2013.

[33] O. A. Arqub, A. el-Ajou, Z. al Zhour, and S. Momani, "Multiple solutions of nonlinear boundary value problems of fractional order: a new analytic iterative technique," Entropy, vol. 16, no. 1, pp. 471-493, 2014.

[34] A. El-Ajou, O. A. Arqub, and S. Momani, “Approximate analytical solution of the nonlinear fractional KdV-Burgers equation: a new iterative algorithm," Journal of Computational Physics, vol. 293, pp. 81-95, 2015.

[35] O. A. Arqub, A. El-Ajou, and S. Momani, "Constructing and predicting solitary pattern solutions for nonlinear time-fractional dispersive partial differential equations," Journal of Computational Physics, vol. 293, pp. 385-399, 2015.

[36] M. Alquran, "Analytical solutions of fractional foam drainage equation by residual power series method," Mathematical Sciences, vol. 8, no. 4, pp. 153-160, 2014.

[37] S. D. Lin and C. H. Lu, "Laplace transform for solving some families of fractional differential equations and its applications," Advances in Difference Equations, vol. 2013, no. 1, 2013.

[38] M. Alquran, "Analytical solution of time-fractional twocomponent evolutionary system of order 2 by residual power series method," Journal of Applied Analysis \& Computation, vol. 5, no. 4, pp. 589-599, 2015.

[39] J. A. Machado, D. Baleanu, W. Chen, and J. Sabatier, New Trends in Fractional Dynamics, SAGE publisher, 2014.

[40] D. Baleanu, Z. B. Güvenç, and J. A. Tenreiro Machado, Eds., New Trends in Nanotechnology and Fractional Calculus Applications, Springer, New York, NY, USA, 2010.

[41] S. Maitama and W. Zhao, "New integral transform: Shehu transform a generalization of Sumudu and Laplace transform 
for solving differential equations," 2019, https://arxiv.org/abs/ 1904.11370.

[42] A. Bokhari, "Application of Shehu transform to AtanganaBaleanu derivatives," Journal of Mathematics and Computer Science, vol. 20, no. 2, pp. 101-107, 2019.

[43] R. Belgacem, D. Baleanu, and A. Bokhari, "Shehu transform and applications to Caputo-fractional differential equations," International Journal of Analysis and Applications, vol. 17, no. 6, pp. 917-927, 2019. 